\title{
Improved Fuzzy C-Mean Algorithm for Image Segmentation
}

\author{
Hind Rustum Mohammed \\ CS dept. Faculty of Computer Science \\ and Mathematics \\ University of Kufa \\ Najaf, Iraq
}

\author{
Husein Hadi Alnoamani \\ MS dept. Faculty of Computer Science \\ and Mathematics \\ University of Kufa \\ Najaf, Iraq
}

\author{
Ali AbdulZahraa Jalil / M.Sc. \\ Student \\ CS dept. Faculty of Computer Science \\ and Mathematics \\ University of Kufa, Najaf, Iraq
}

\begin{abstract}
The segmentation of image is considered as a significant level in image processing system, in order to increase image processing system speed, so each stage in it must be speed reasonably. Fuzzy c-mean clustering is an iterative algorithm to find final groups of large data set such as image so that is will take more time to implementation. This paper produces an improved fuzzy c-mean algorithm that takes less time in finding cluster and used in image segmentation.
\end{abstract}

Keywords-pattern recognition; image segmentation; fuzzy cmean; improved fuzzy c-mean; algorithms

\section{INTRODUCTION}

To recognize pattern and analysis an image the main process is segmentation of image[1-3]. Is an operation of dividing an image into parts that have same features and the collection of these parts form the original image[4]. Fig.1. illustrate variant levels of processing of image and technique of analyzing [5], and it shows clearly segmentation stage.

There are many types of image's pattern recognition and segmentation, but there are two mainly types of classification which are used: Supervised classification and unsupervised classification, in the first one the classes are defined in advance and in the second they are not defined in advance which known as clustering. There are two types of clustering: hard clustering and fuzzy clustering, in hard clustering, the data item is belong exactly to one cluster but in fuzzy clustering, the data item belong by the degree of membership to each cluster of clusters, and the summation of all memberships values to one of data items is equal to one.

Fuzzy c-mean clustering is one of unsupervised clustering algorithms that is widely used in image processing and computer vision because it easy to implement and clustering performance[6], [7]. It's used to segment an image by grouping pixels that have similar or nearly similar values into a cluster, where each group of pixel's values that belong to one cluster are similar to each other and different from pixel's values that belong to other clusters, and then these clusters represent the segments of the segmented image. The traditional fuzzy cmean suffers from some limitations, it's not accurate in the segmentation of noisy image and time consuming because it's iterative nature. Our proposed algorithm which named Improved fuzzy c-mean algorithm offers an overcoming of one limitation of traditional fuzzy c-mean which is timeconsuming.

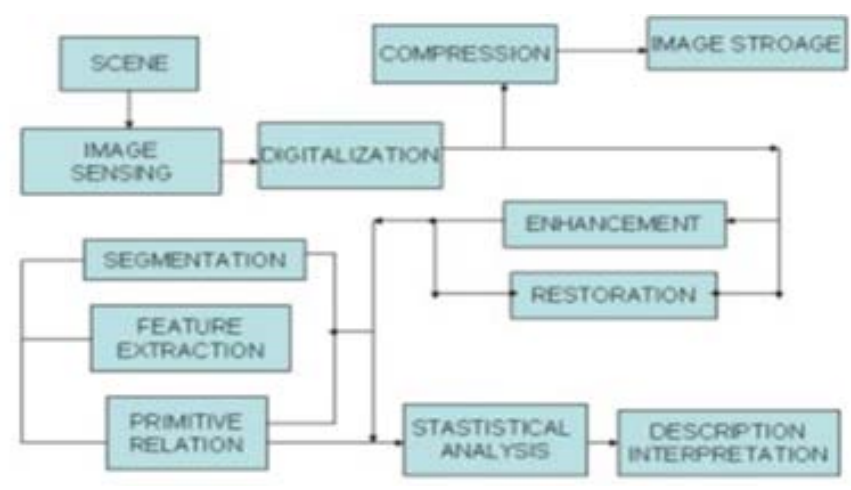

Fig. 1. Schema of variant levels of processing of image and technique of analyzing

In our proposed algorithm we use frequency of each data item of image and processing these frequencies instead of processing whole data items of the image. That is reduce processing time in the great form. This paper contains five parts and arranged as follow: Section 2 talking about time complexity, section 3 the traditional fuzzy c-mean, section 4 proposed an algorithm, section 5 Experimental Results, and in section 6 the conclusion.

\section{TIME COMPLEXITY}

It's time that required to run or execute an algorithm[11].the notation big $\mathrm{O}$ is used to express time complexity. it's proportional to the size of input data. If the input size is $n$, then the time complexity is the time required by the algorithm to process these input. Each algorithm has a primitive operation(s), so the time of the algorithm is determined by computing the summation of times that required to run each of these operations. It's always expressed by the prevalent term, which is the term have exponent with the highest value. It also ignores constant of multiplication and constant of the division. if the time required to accomplish an algorithm of $n$ input size is $10 n^{3}+6 n$, then the expression of its time complexity is $O\left(n^{3}\right)$ also if the time required to accomplish an algorithm is $\mathrm{c}^{*} \mathrm{n}^{2}$ or $\mathrm{n}^{2} / \mathrm{c}$ where $\mathrm{c}$ is constant then the time complexity is $\mathrm{O}\left(\mathrm{n}^{2}\right)$. If the algorithm processed all inputted data to get the desired solution, then the time complexity called the worst-case of time complexity, which is show, the algorithm take maximum time to achieve the required process. There are many types of time complexities which depend on algorithm's function nature. Some common types of time complexities are constant time 
$\mathrm{O}(1)$, linear time $\mathrm{O}(\mathrm{n})$, quadratic time $\mathrm{O}\left(\mathrm{n}^{2}\right)$, exponential time $\mathrm{O}\left(\mathrm{c}^{\mathrm{n}}\right)$ where $\mathrm{c} \geq \mathrm{n}>1$. In our proposed algorithm we suggest an algorithm that consumes so little time amount compared with the traditional fuzzy C-mean algorithm.

\section{TRADITIONAL FUZZY C-MEAN}

The fuzzy c-mean algorithm is one of the common algorithms that used to image segmentation by dividing the space of image into various cluster regions with similar image's pixels values. For medical images segmentation, the suitable clustering type is fuzzy clustering. The Fuzzy c-means (FCM) can be seen as the fuzzified version of the k-means algorithm. It is a clustering algorithm which enables data item to have a degree of belonging to each cluster by degree of membership. It's developed by Dunn [9] and changed by Bezdek [10]. The algorithm is an iterative clustering method that produces an optimal c partition by minimizing the weighted within group sum of squared error objective function [10]. Is widely used in image segmentation and pattern recognition. Following are steps of traditional fuzzy c-mean:

Step1:Choose random centroid at least 2 and put values to them randomly.

Step2:Compute membership matrix:

$$
\mathrm{U}_{\mathrm{ij}}=\frac{1}{\sum_{\mathrm{k}=1}^{\mathrm{c}}\left[\frac{\left|\mathrm{x}_{\mathrm{i}}-\mathrm{c}_{\mathrm{j}}\right|}{\mathrm{x}_{\mathrm{i}}-\mathrm{c}_{\mathrm{k}}}\right]^{\frac{2}{\mathrm{~m}-1}}} \text {, where } \mathrm{m}>1 \text {, c cluster's No. }
$$

Step3: calculate the clusters centers:

$$
\mathrm{C}=\frac{\sum_{\mathrm{i}=1}^{\mathrm{n}} \mathrm{U}^{\mathrm{m}}{ }_{\mathrm{ij}} \mathrm{x}_{\mathrm{i}}}{\sum_{\mathrm{i}=1}^{\mathrm{n}} \mathrm{U}^{\mathrm{m}_{\mathrm{ij}}}}
$$

Step4: if $C^{(\mathrm{k}-1)}-\mathrm{C}^{\mathrm{k}}<\varepsilon$ then Stop else go to Step2.

This traditional algorithm is an iterative algorithm that suffers from time and memory consuming because it computes membership value for each item in the data.

\section{PROPOSED AlgORITHM}

In the following section we provide the improved fuzzy cmean algorithm:

Step1: Let $\mathrm{H}$ represent the frequency of each item in Data.

Step2:create vector $I=\min ($ Data $): \max ($ Data $)$

Step3:Choose random centroid at least 2.

Step4:Compute membership matrix:

$$
\mathrm{U}_{\mathrm{ij}}=\frac{1}{\sum_{\mathrm{k}=1}^{\mathrm{c}}\left[\frac{\left|\mathrm{I}_{\mathrm{i}}-\mathrm{c}_{\mathrm{j}}\right|}{\left|\mathrm{I}_{\mathrm{i}}-\mathrm{c}_{\mathrm{k}}\right|}\right]^{\frac{2}{\mathrm{~m}-1}}}
$$

Step5: calculate the cluster center:

$$
\mathrm{C}=\frac{\sum_{\mathrm{i}=1}^{\mathrm{n}} \mathrm{U}^{\mathrm{m}} * \mathrm{H} * \mathrm{I}}{\sum_{\mathrm{i}=1}^{\mathrm{n}} \mathrm{U}^{\mathrm{m}} * \mathrm{H}}
$$

Step6: if $\mathrm{C}^{(\mathrm{k}-1)}-\mathrm{C}^{\mathrm{k}}<\varepsilon$ then Stop else go to Step4.

The improved fuzzy c-mean use values that represent the frequency of items instead of actual values, in gray images the number of values of it may be reached to $256 * 256=65,536$ and that is will take more time in processing, but in improved algorithm will take, at worst case, 256 item to process it. The proposed algorithm does not depend on whole data of image, it actually depends on data that represent the frequency of each data item in original image's data. A number of frequencies at most is 256 .

\section{EXPERIMENTS RESULTS}

We tested Improved fuzzy c-mean by implemented by using MATLAB and compared it with implementation of fuzzy c-mean algorithm that used by MATLAB by calling command fcm, we try algorithm in database of images contains 100 images, in the following we provide a sample from tested images, in this testing sample we use $\mathrm{C}=3$ :

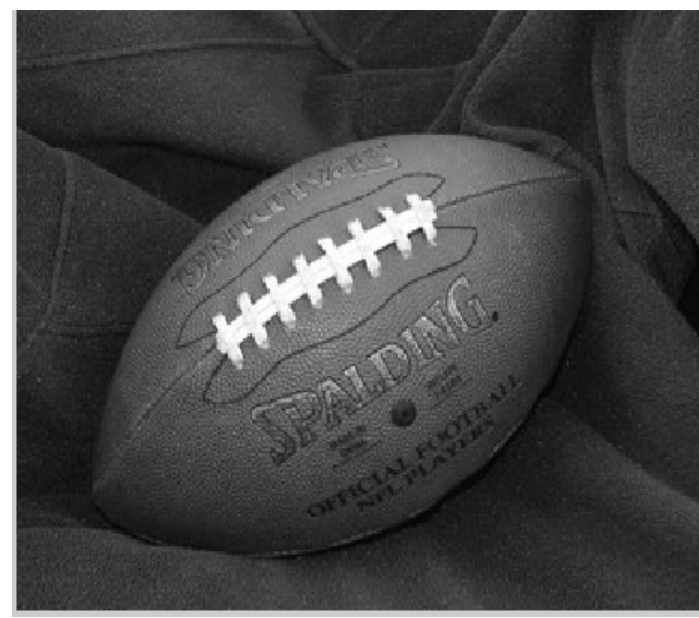

Fig. 2. Orginal image, "football”

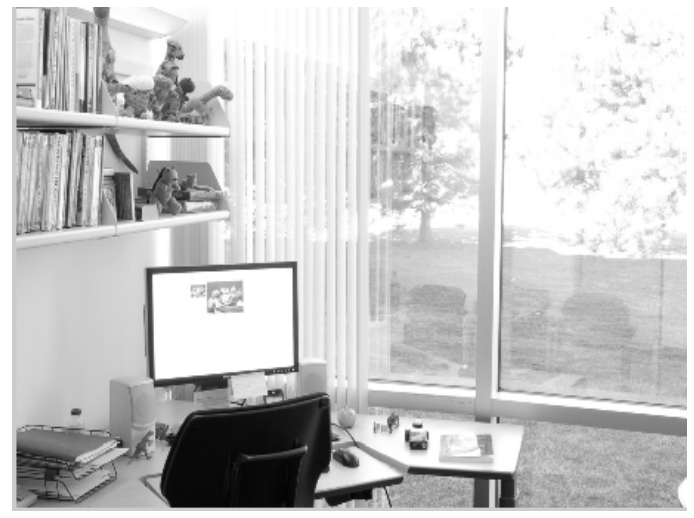

Fig. 3. Orginal image, "office" 


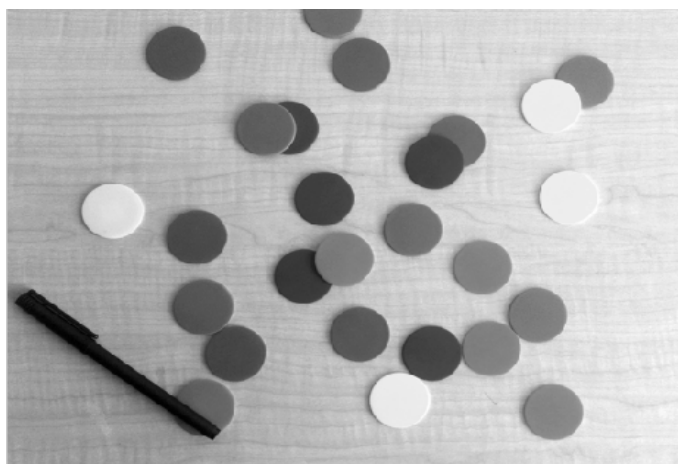

Fig. 4. Orginal image, “coloredChips”

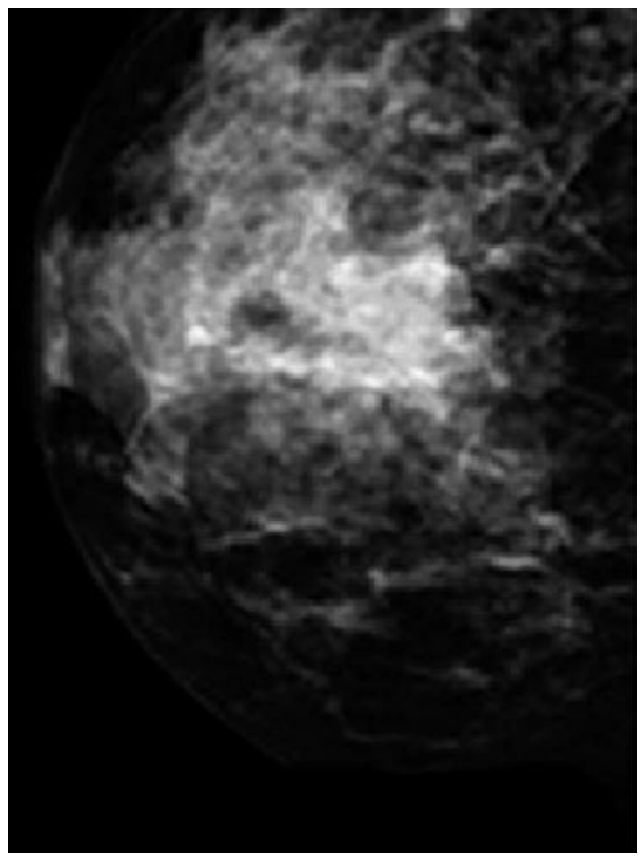

Fig. 5. Orginal image, "breast”

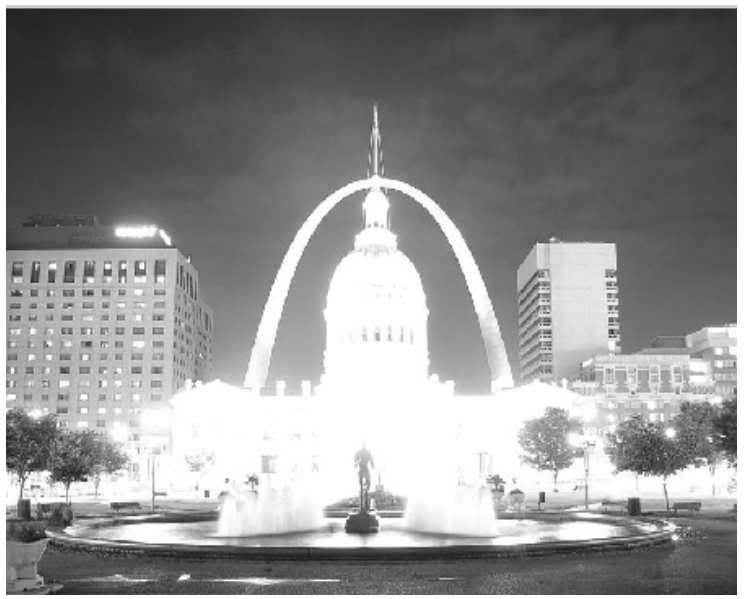

Fig. 6. Orginal image, "house" segmented image by fom

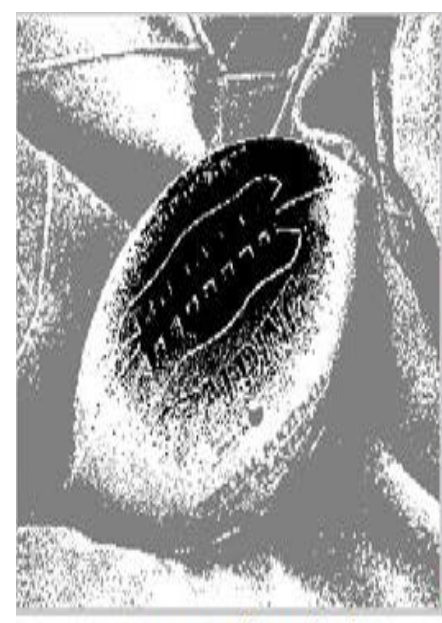

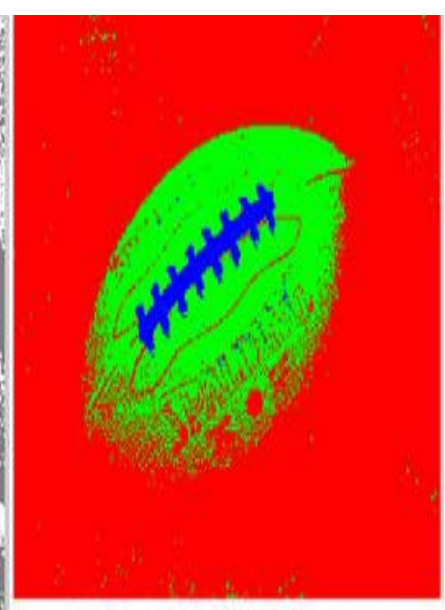

segmented by proposed fom
Fig. 7. Comparison between fcm and proposed fcm on "football” image

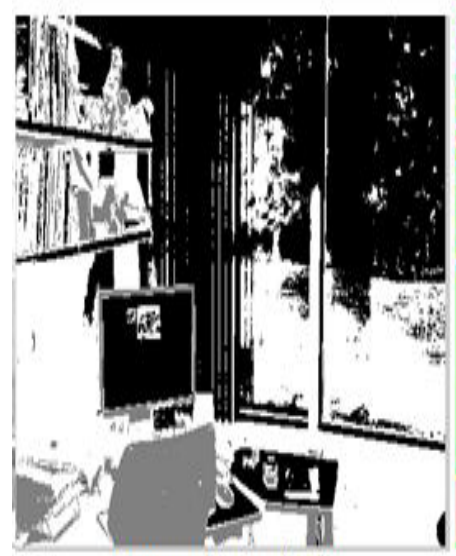

segmented image by fcm

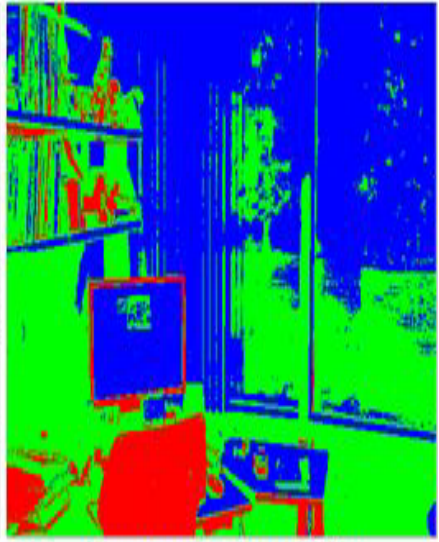

segmented by proposed fcm
Fig. 8. Comparison between fcm and proposed fcm on "office” image

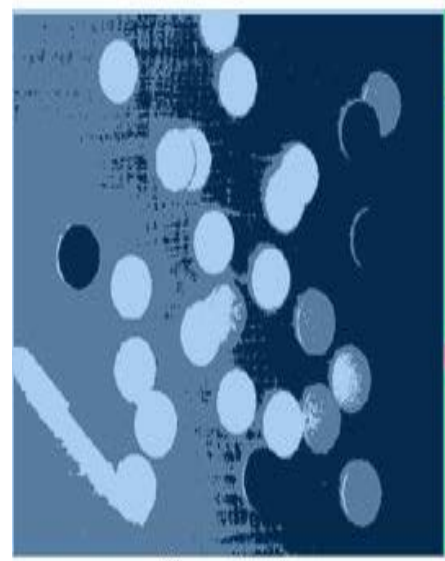

segmented image by $\mathrm{fcm}$

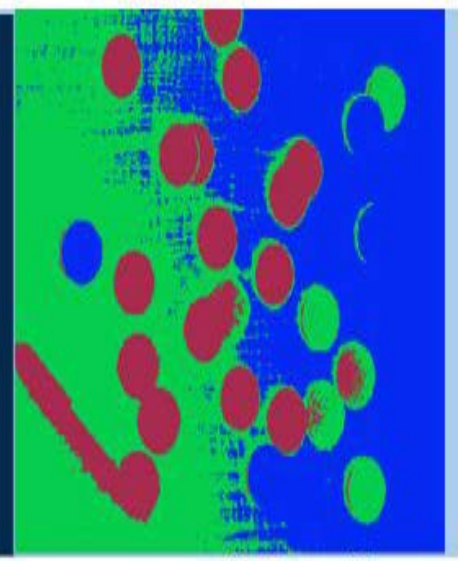

segmented by proposed fcm
Fig. 9. Comparison between fcm and proposed fcm on "coloredChips" image 


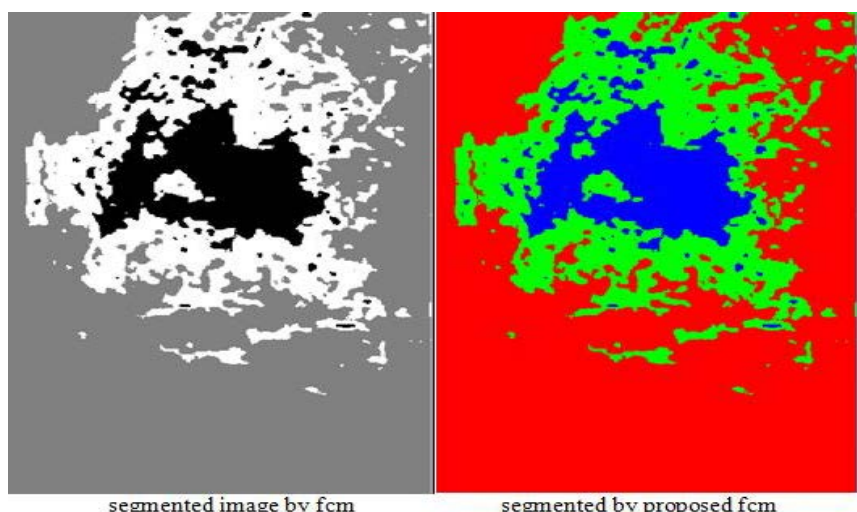

Fig. 10. Comparison between fcm and proposed fcm on "breast" image

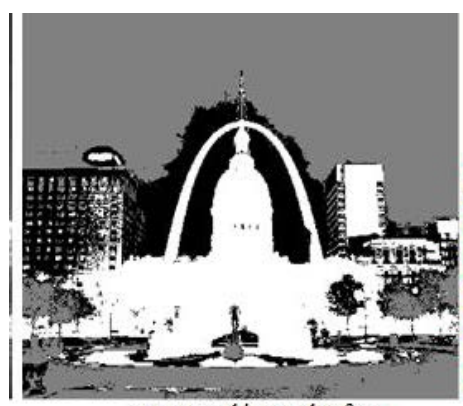

segmented image by fom

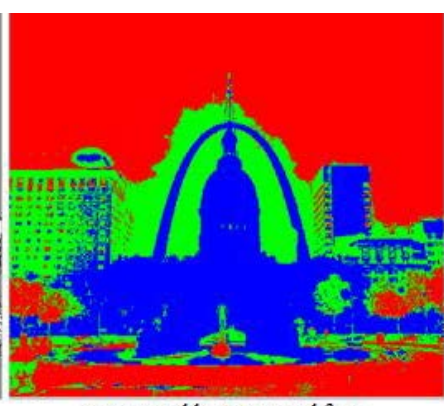

segmented by proposed $\mathrm{fcm}$
Fig. 11. Comparison between fcm and proposed fcm on "house" image

TABLE I. TIME COMPARISON BETWEEN TRADITIONAL FCM AND PROPOSED FCM

\begin{tabular}{|l|l|l|}
\hline Image name & $\begin{array}{l}\text { Segmentation time by } \\
\text { fcm }(\mathbf{s e c})\end{array}$ & $\begin{array}{l}\text { Segmentation time by } \\
\text { proposed fcm (sec) }\end{array}$ \\
\hline football & 3.828125 & 0.015625 \\
\hline office & 22.796875 & 0.140625 \\
\hline coloredChips & 8.296875 & 0.031250 \\
\hline breast & 14.00000 & 0.062500 \\
\hline house & 24.828125 & 0.078125 \\
\hline
\end{tabular}

\section{CONCLUSION}

From above results in accuracy and speed of proposed fuzzy c-mean algorithm compared with the traditional fuzzy cmean algorithm, we conclude this algorithm is a great enhancement in implementation and performance of traditional fuzzy c-mean.

\section{REFERENCES}

[1] K.S. Deshmukh, G.N. Shinde, An adaptive color image segmentation, Electron.Lett. Comput. Vis. Image Anal. 5 (4) (2005) 12-23.

[2] Y. Zhang, A survey on evaluation methods for image segmentation, Pattern Recognition 29 (8) (1996) 1335-1346.

[3] V. Boskovitz, H. Guterman, An adaptive neuro-fuzzy system for automaticy image segmentation and edge detection, IEEE Trans. Fuzzy Syst. 10 (2) (2002) 247-262.

[4] C.Harris and M.Stephens, "A Combined Corner and Edge Detection," Proc.Fourth Alvey Vision Conf., pp.147-151, 1988.

[5] Cahoon, T.C .Sutton, M .A. Bezdek "Brest cancer detection using image processing techniques”, J.C.Dept.of Comp.Sci.Univ.of West Florida, Pensacola, FL Fuzzy IEEE 2000.The ninth IEEE conference.

[6] D. L. Pham and J. L. Prince, "An adaptive fuzzy c-means algorithm for image segmentation in the presence of intensity in homogeneities," Pattern Recognition. Lett. vol. 20, pp 57-68, 1999.

[7] W. J. Chen, M. L. Giger, and U. Bick, “A fuzzy c-means (FCM)-based approach for computerized segmentation of breast lesions in dynamic contrast enhanced MRI images,” Acad. Radiol, vol. 13, pp. 63-72, 2006.

[8] J. M. Gorriz, J. Ramirez, E. W. Lang, and C. G. Puntonet, "Hard cmeans clustering for voice activity detection,” Speech Commun, vol. 48, pp. 1638-1649, 2006

[9] J. C. Dunn, “A fuzzy relative of the ISODATA process and its use in detecting compact well-separated clusters,” J. Cybernetics, vol. 3, no. 3,pp. 32-57, 1973.

[10] J. C. Bezdek, "Pattern recognition with fuzzy objective function algorithms,” New York, Plenum, 1981.

[11] Sipser, Michael, "Introduction to the Theory of Computation". Course Technology Inc. ISBN 0-619-21764-2, 2006. 